\title{
Viabilidade financeira de sistemas agroflorestais biodiversos no Centro Oeste
}

\section{Brasileiro}

\author{
Financial viability of biodiverse agroforestry systems in the Brazilian Midwest \\ Viabilidad financiera de los sistemas agroforestales biodiversos en el Medio Oeste brasileño
}

Recebido: 09/03/2021 | Revisado: 26/03/2021 | Aceito: 09/04/2021 | Publicado: 18/04/2021

\author{
Leila Tatiana Garcia \\ ORCID: https://orcid.org/0000-0002-1544-4080 \\ Secretaria Municipal de Educação de Campo Grande, Brasil \\ E-mail: leilatatig@gmail.com \\ Landi Arami Rosato Paulus \\ ORCID: https://orcid.org/0000-0002-1123-6831 \\ Jacarandá Consultoria e Assessoria Ambiental, Brasil \\ E-mail: landirossato@gmail.com \\ Shaline Séfara Lopes Fernandes \\ ORCID: https://orcid.org/0000-0001-8525-404X \\ Universidade Estadual de Mato Grosso do Sul, Brasil \\ E-mail: shaline_sefara@ hotmail.com \\ Marcelo Francia Arco-Verde \\ ORCID: https://orcid.org/0000-0001-6545-9961 \\ Embrapa Florestas, Brasil \\ E-mail: marcelo.arco-verde@embrapa.br \\ Milton Parron Padovan \\ ORCID: https://orcid.org/0000-0002-7888-1915 \\ Empresa Brasileira de Pesquisa Agropecuária Oeste, Brasil \\ E-mail: milton.padovan@embrapa.br \\ Zefa Valdivina Pereira \\ ORDID: https://orcid.org/0000-0001-5328-2169 \\ Universidade Federal da Grande Dourados, Brasil \\ E-mail: zefapereira@ufgd.edu.br
}

\begin{abstract}
Resumo
Os sistemas agroflorestais biodiversos (SAF's) combinam espécies arbóreas frutíferas ou lenhosas, com culturas agrícolas e/ou criação de animais, em uma mesma área. Para o agricultor que o detém, esses sistemas trazem melhoria da qualidade de vida econômica, social e ambiental, pois proporcionam formas sustentáveis de produção de alimentos, segurança alimentar, além da preservação ambiental. O estudo apresenta a análise de viabilidade financeira de dois modelos de sistemas agroflorestais implantados no município de Dourados, MS. A pesquisa objetivou fornecer subsídios aos agricultores familiares para a implementação de SAF's. As culturas de interesse econômico selecionadas para composição dos SAF's implantados foram: SAF1- café, abacaxi, milho, feijão; SAF2 - palmito de pupunha, banana, abacaxi, milho, feijão, além de nove espécies arbóreas para ambos os SAF's: acerola, graviola, goiaba, pitanga, aroeira, guaritá, ipê, canafístula e peito de pombo, cada SAF's contou com um hectare. Para planejamento e elaboração dos sistemas, foi utilizada a planilha AmazonSAF, onde foram inseridos dados de receitas e despesas, que refletem a realidade da agricultura familiar, verificando a rentabilidade ao longo de 20 anos. Ambos os SAF's apresentaram condições de viabilidade financeira, pois o Valor Presente Líquido do SAF 1 foi de R $\$ 11.018,24$ e do SAF 2 foi de $R \$ 40.377,04$. Este último se apresentou ligeiramente como o mais rentável, já que sua relação Benefício/Custo foi de 1,2 e o payback 9 anos, diferente do SAF 1, cuja RB/C foi de 1,1 e payback de 10 anos. A viabilidade financeira, tanto do SAF 1, com café como cultura principal, quanto do SAF 2, com palmito de pupunha e banana, foi confirmada, de forma a garantir ao produtor rural que, se bem planejados e acompanhados, estes sistemas demonstraram boas condições para produção de alimentos e geração de renda. Além disso, se tornam soluções possíveis para uma agricultura sustentável, valorizando e utilizando sabiamente os recursos naturais.
\end{abstract}

Palavras-chave: Agroecologia; Agrofloresta; Sustentabilidade.

\section{Abstract}

The biodiverse agroforestry systems (BAS) combine fruitful or woody tree species, with agricultural crops and / or animal husbandry, in the same area. For the farmer who owns it, these systems improve the quality of economic, social and environmental life, as they provide sustainable forms of food production, food security, in addition to environmental preservation. The study presents the financial viability analysis of two models of agroforestry systems 
implemented in the municipality of Dourados, MS. The research aimed to provide subsidies to family farmers for the implementation of SAF's. The crops of economic interest selected for the composition of the implanted SAF's were: BAS1- coffee, pineapple, corn, beans; BAS2 - pupunha palm, banana, pineapple, corn, beans, in addition to nine tree species for both BAS: acerola, soursop, guava, pitanga, aroeira, guaritá, ipê, canafístula and pigeon breast, each BAS had one hectare . For planning and elaboration of the systems, the AmazonSAF spreadsheet was used, where data of income and expenses were inserted, which reflect the reality of family farming, verifying profitability over 20 years. Both BAS was financially viable, as the Net Present Value of BAS 1 was R\$ 11,018.24 and BAS 2 was R\$ 40,377.04. The latter presented itself slightly as the most profitable, since its Benefit/Cost ratio was 1.2 and the payback was 9 years, different from BAS 1, whose RB/C was 1.1 and the payback was 10 years. The financial viability of both BAS 1 , with coffee as the main crop, and BAS 2, with pupunha and banana hearts, was confirmed, in order to guarantee to the rural producer that, if well planned and monitored, these systems showed good conditions for food production and income generation. In addition, they become possible solutions for sustainable agriculture, valuing and using natural resources wisely.

Keywords: Agroecology; Agroforestry; Sustainability.

\section{Resumen}

Los sistemas agroforestales biodiversos (SAF) combinan especies de árboles frutales o leñosos, con cultivos agrícolas y/o ganadería, en la misma zona. Para el agricultor que lo posee, estos sistemas mejoran la calidad de vida económica, social y ambiental, ya que brindan formas sustentables de producción de alimentos, seguridad alimentaria, además de la preservación ambiental. El estudio presenta el análisis de viabilidad financiera de dos modelos de sistemas agroforestales implementados en la ciudad de Dourados, MS. La investigación tuvo como objetivo otorgar subsidios a los agricultores familiares para la implementación de los SAF. Los cultivos de interés económico seleccionados para la composición de los SAF implantados fueron: SAF 1- café, piña, maíz, frijol; SAF 2 - palma pupunha, plátano, piña, maíz, frijoles, además de nueve especies de árboles para ambos SAF: acerola, guanábana, guayaba, pitanga, aroeira, guaritá, ipê, canafístula y pechuga de paloma, cada SAF tenía una hectárea. Para la planificación y elaboración de los sistemas se utilizó la hoja de cálculo de AmazonSAF, donde se insertaron datos de ingresos y egresos, que reflejan la realidad de la agricultura familiar, verificando la rentabilidad a más de 20 años. Ambos SAF eran financieramente viables, ya que el Valor Actual Neto del SAF 1 era de R\$11.018,24 y el del SAF 2 era de R\$ 40.377,04. Este último se presentó levemente como el más rentable, ya que su relación Beneficio/Costo fue de 1,2 y la recuperación fue de 9 años, diferente del SAF 1, cuyo RB / C fue de 1,1 y la recuperación fue de 10 años. Se confirmó la viabilidad financiera tanto del SAF 1, con café como cultivo principal, como del SAF 2, con pupunha y corazones de banano, a fin de garantizar al productor rural que, bien planificados y monitoreados, estos sistemas presentaban buenas condiciones para producción de alimentos y generación de ingresos. Además, se convierten en posibles soluciones para la agricultura sostenible, valorando y utilizando sabiamente los recursos naturales.

Palabras clave: Agroecología; Agroforestería; Sustentabilidad.

\section{Introdução}

Os Sistemas Agroflorestais caracterizam-se pelo cultivo de plantas lenhosas perenes (árvores, arbustos, bambu, etc.) combinadas com culturas agrícolas e/ou animais, em mesma unidade de terra de forma simultânea ou em sequência temporal, interagindo econômica e ecologicamente (Nair, 1993; Sharma, 2013; Silva, 2013; Nair, 2014). O termo "biodiversos" foi cunhado por Peneireiro (2003) para caracterizar os agroecossistemas mais complexos e que buscam os fundamentos da floresta em seus princípios ecológicos (Camargo et al. 2019; Padovan et al., 2019).

Estes sistemas fornecem diversos serviços ecossistêmicos como a estabilidade microclimática, ciclagem de nutrientes, diminuição da erosão, redução de salinidade e acidez, diminuição da demanda de insumos externos, melhoramento na infiltração de água no solo, recuperação da fertilidade do solo, aumento da diversidade de inimigos naturais, de polinizadores e da biota do solo; sequestro de carbono na biomassa vegetal; retorno da fauna ao sistema; produção local de grande quantidade de materiais orgânicos, além da capacidade de recompor, a longo prazo, a vegetação nativa por meio de espécies arbóreas e arbustivas (Padovan, 2014; Mosquera-Losada et al., 2016; Torralba et al., 2016; Zomer et al., 2016; Coelho, 2017; Udawatta et al., 2019; Sousa et al., 2020).

Configuram-se como alternativas à agricultura sustentável devido a diversificação da produção e a otimização no uso da terra (Molua, 2005; Paludo \& Costabeber 2012). Esses sistemas produzem boa diversidade de alimentos, sendo estratégicos à segurança alimentar e nutricional das famílias agricultoras, pois gera renda e vários benefícios sociais, ao mesmo tempo que 
desempenha as funções ecológicas (Miccolis et al., 2017).

Devido aos seus benefícios ambientais e socioeconômicos, os sistemas agroflorestais biodiversos revestem-se de grande importância para a agricultura familiar e à sociedade em geral (Camargo et al., 2019; Martinelli et al., 2019). Contudo, apesar dos avanços nos estudos com esses agroecossistemas, poucos avaliam a viabilidade financeira de diferentes arranjos de espécies vegetais (Arco-Verde \& Amaro 2014, 2015; Camargo, 2019; Martinelli et al., 2019). Essas análises possibilitam aprimoramentos no planejamento e gerenciamento da unidade rural, evitando gastos desnecessários e aumentando a rentabilidade dos agricultores (Arco-Verde \& Amaro, 2015).

Com essas análises, os agricultores poderão estabelecer critérios mais eficazes para alcançar resultados satisfatórios na produção rural (Nasser et al., 2012), pois, segundo Silva (2013), já foi largamente comprovado o potencial dos SAFs, mas os agricultores precisam mais do que isso. Ou seja, necessitam que esse potencial produtivo e gerador de renda, bem como de serviços ambientais, sejam levados à realidade rural, como algo sustentável no uso da terra.

Nesse sentido, desenvolveu-se este trabalho com intuito de analisar a viabilidade financeira de dois arranjos agroflorestais visando subsidiar aos agricultores e técnicos em tomadas de decisões para implementação desses agroecossistemas na macrorregião Centro Oeste do Brasil.

\section{Metodologia}

\section{Área experimental}

Os sistemas agroflorestais avaliados (SAFs 1 e 2) foram implantados na Fazenda Experimental de Ciências Agrárias da Universidade Federal da Grande Dourados - UFGD, no Km 20, próximo à BR 463 Dourados - Ponta Porã, no município de Dourados, Estado de Mato Grosso do Sul, no Centro Oeste do Brasil. O local do experimento situa-se entre as coordenadas O 55 00' 09" / S 22 15' 03" e O 54 59' 02" / S 22 13' 18" e altitude de 434 m. O clima da região e considerado de transição entre o tropical e o subtropical e segundo a classificação de Koppen e do tipo Aw úmido com inverno seco e verão chuvoso, com precipitação média anual de $1.410 \mathrm{~mm}$ (Arai et al., 2010). O solo é classificado como Latossolo Vermelho Distrófico, textura argilosa (Embrapa, 2006). A formação florestal da Fazenda Experimental de Ciências Agrárias caracteriza-se como Floresta Estacional Semidecidual Submontana (IBGE, 2012).

\section{Implantação dos Sistemas Agroflorestais}

Foi estabelecida uma área de $10.000 \mathrm{~m}^{2}$ para cada um dos dois arranjos agroflorestais. Inicialmente, o solo foi preparado por meio de gradagens para eliminação de plantas espontâneas. A implantação dos SAFs ocorreu nos meses de janeiro e fevereiro de 2016. Foram coletados dados de produtividade para o primeiro ano em campo (2017), e realizado uma análise financeira representada por simulações para um período de 20 anos (2017 a 2037).

Foram selecionadas nove espécies arbóreas, considerando seu potencial para comercialização de frutos e a adaptabilidade desta a região. Estas foram implantadas em 10 linhas visando a melhoria da biodiversidade no agroecossistema, como a atração da fauna, além da potencialização de outros serviços ambientais, favorecendo a obtenção de resultados positivos, tanto econômicos quanto ecológicos e biológicos (Silva, 2013).

O espaçamento nas entrelinhas das arbóreas foi de $11 \mathrm{~m}$, e nas bordas $(0,5 \mathrm{~m}$ de cada lado) foram implantados Canavalia ensiformes (feijão de porco) e Cajanus cajan (feijão guandu) como adubação verde e sistema de quebra-vento para proteção, respectivamente.

Em cada linha de arbóreas e arbustivas foram plantadas 34 mudas aleatórias, com espaçamento de $3 \mathrm{~m}$ entre plantas e $11 \mathrm{~m}$ entre linhas, totalizando 340 mudas de espécies frutíferas e lenhosas. 
Nas entrelinhas das árvores e arbustos, foram implantados cafeeiros, abacaxizeiros, feijoeiros e milho no SAF 1, bem como bananeiras, pupunheiras, abacaxizeiros, milho e feijoeiros no SAF 2 (Figuras 1 A e 1B).

Figura 1 - Croqui dos Sistemas Agroflorestais implantados na Fazenda Experimental de Ciências Agrárias da Universidade Federal da Grande Dourados, em Dourados, MS: A - SAF 1; B - SAF 2. (Ambos demonstram apenas uma entrelinha).

A

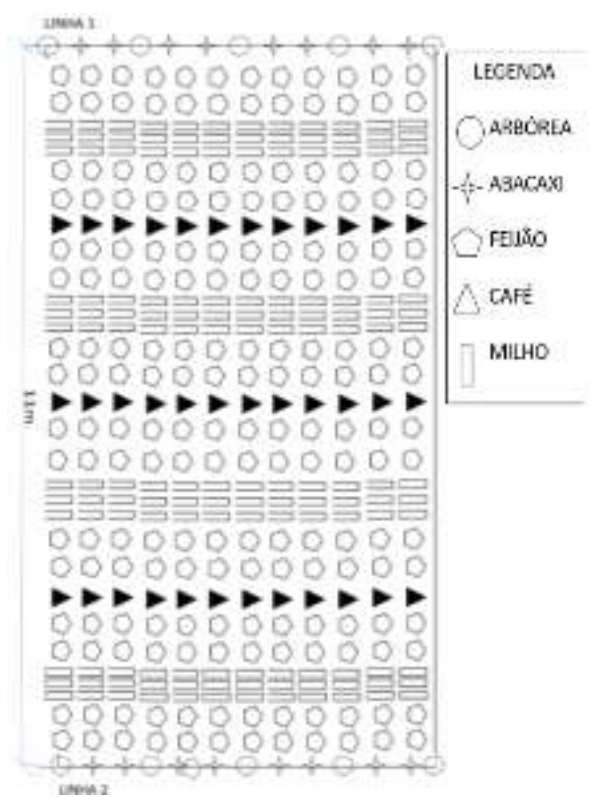

B

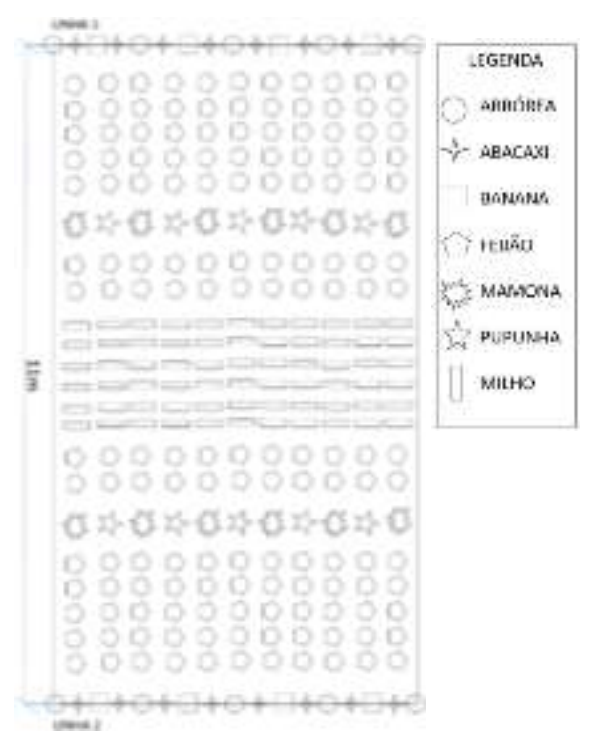

Fonte: Autores.

Na Figura 1, está representado o esquema de plantio das espécies no SAF 1 e SAF 2.

As espécies destinadas à produção para gerar renda (café, palmito de pupunha, banana, abacaxi, milho e feijão) foram selecionadas considerando a adaptação às condições edafoclimáticas da região, além da possibilidade de mercado, principalmente nas feiras agroecológicas, entre outros canais de comercialização. O produto principal para a comercialização é o café no SAF 1, enquanto no SAF 2 é o palmito de pupunha. A Tabela 1 apresenta a descrição das características principais dos componentes dos sistemas agroflorestais, além do espaçamento e densidade dentro dos agroecossistemas. 
Tabela 1 - Espécies arbóreas nativas e de interesse econômico selecionadas para compor os Sistemas Agroflorestais em Dourados, MS 2016.

\begin{tabular}{|c|c|c|c|c|c|c|c|c|}
\hline Nome Científico & Família & Nome popular & Grupo & Estrato & $\begin{array}{l}\text { Ciclo de vida } \\
\text { (Aproximado) }\end{array}$ & $\begin{array}{l}\text { Espaçamento } \\
\text { (metros) }\end{array}$ & $\begin{array}{c}\text { Densidade } \\
\text { SAF 1 } \\
\left(\text { plantas.ha }{ }^{-1}\right) \\
\end{array}$ & $\begin{array}{c}\text { Densidade } \\
\text { SAF 2 } \\
\left(\text { plantas.ha }{ }^{-1}\right)\end{array}$ \\
\hline Peltophorum dubium (Spreng.) Taub. & Fabaceae & Canafístula & Arbórea & Alto & Perene & $3 \times 11$ & 37 & 37 \\
\hline $\begin{array}{l}\text { Handroanthus heptaphyllus (Vell.) } \\
\text { Mattos }\end{array}$ & Bignoniaceae & Ipê rosa & Arbórea & Alto & Perene & $3 \times 11$ & 38 & 37 \\
\hline Tapirira guianensis Aubl. & Anacardiaceae & Peito de pombo & Arbórea & Alto & Perene & $3 \times 11$ & 37 & 38 \\
\hline Myracrodruon urundeuva Allemão & Anacardiaceae & Aroeira-da-serra & Arbórea & Alto & Perene & $3 \times 11$ & 38 & 38 \\
\hline Astronium graveolens Jacq. & Anacardiaceae & Guaritá & Arbórea & Alto & Perene & $3 \times 11$ & 38 & 38 \\
\hline Eugenia uniflora L. & Myrtaceae & Pitanga & Arbórea & Alto & Perene & $3 \times 11$ & 38 & 38 \\
\hline Psidium guajava $\mathrm{L}$. & Myrtaceae & Goiaba & Arbustiva & Médio & Perene & $3 \times 11$ & 38 & 38 \\
\hline Malpighia emarginata DC. & Malpighiaceae & Acerola & Arbustiva & Médio & Perene & $3 \times 11$ & 38 & 38 \\
\hline Annona muricata $\mathrm{L}$. & Annonaceae & Graviola & Arbustiva & Médio & Perene & $3 \times 11$ & 38 & 38 \\
\hline Coffea arabica cv. IAPAR 59 & Rubiaceae & Café & Agrícola & Baixo & Perene & $1 \times 5$ & 2.646 & --- \\
\hline Bactris gasipaes Kunth & Arecaceae & Pupunha & Agrícola & Médio & Perene & $1 \times 5$ & --- & 1.764 \\
\hline Musa spp. Cv. nanicão & Musaceae & Banana & Agrícola & Médio & 15 anos & $3 \times 11$ & --- & 330 \\
\hline Ananas comosus (L.) Merril & Bromeliaceae & Abacaxi & Agrícola & Baixo & 2 anos & $0,4 \times 1$ & 2.310 & 1.980 \\
\hline Zea mays L. & Poaceae & Milho & Agrícola & Baixo & Anual & $0,2 \times 1$ & 10.584 & 4.410 \\
\hline Phaseolus vulgaris L. & Fabaceae & Feijão & Agrícola & Baixo & Anual & $0,1 \times 0,4$ & 35.280 & 48.510 \\
\hline
\end{tabular}

Fonte: Autores.

Na Tabela 1, consta a diversidade de espécies, as características ecológicas e a densidade de espécies que compõem o SAF 1 e 2. 


\section{Manejo da área implantada}

O manejo consistiu em limpezas manuais e roçadas tratorizadas de plantas espontâneas, em sua maioria Brachiaria sp., deixando seus resíduos sobre o solo para otimizar os processos de troca de energia, pois segundo Götsch (1997), o objetivo é sempre criar mais fertilidade e vida ao solo, excluindo o uso de maquinário pesado, de agrotóxicos ou do fogo para a limpeza do campo. Não foi utilizada capina química, ou seja, substâncias agrotóxicas. Quanto à irrigação, somente foram realizados molhamentos com regadores manuais na implantação das mudas das espécies vegetais.

\section{Análise dos dados}

Para avaliação da viabilidade financeira dos dois arranjos de SAFs, foi utilizado o instrumento AmazonSAF, uma planilha de Avaliação Financeira de Sistemas Agroflorestais, idealizada por Arco-Verde \& Amaro (2010), com intuito de contribuir com os produtores rurais e técnicos em tomadas de decisão. Essa planilha possibilita observar de modo transparente o comportamento do SAF ao longo de 10, 20 ou 30 anos, já que está organizada em abas didáticas e intuitivas tais quais: descrição dos componentes do sistema, croqui, parâmetros técnicos, produtividade dos cultivos, atividades e insumos gerais, guias das culturas, resultado financeiro, fluxo de caixa, indicadores financeiros e gráficos.

Dessa forma, os indicadores de rentabilidade considerados foram: taxa interna de retorno (TIR), valor presente líquido (VPL), tempo para recuperação do capital (payback simples e descontado), valor anualizado equivalente (VAE), relação benefício-custo $(\mathrm{RB} / \mathrm{C})$, além da remuneração da mão de obra familiar (RMOF), considerada $\mathrm{R} \$ 70,00$ segundo o valor médio atual da diária no mercado. A taxa de juros ao ano foi considerada de 6,5\% estabelecido pelo Ministério do Desenvolvimento Agrário para financiamento do PRONAF Agroecologia.

\section{Fontes das informações}

O presente trabalho consistiu em um levantamento descritivo, quantitativo e documental (Pereira et al., 2018). Além das pesquisas na literatura, foram consultados diversos produtores e técnicos que atuam na agroecologia, para darem informações sobre indicadores técnicos, estimativas de produtividade ao longo do tempo e preços de venda, tendo em vista que os SAFs implantados ainda não estavam gerando renda. Os custos referentes à implantação dos SAFs, compreendendo: mudas, sementes, manutenção, transporte, diárias, entre outras, foram obtidos diretamente com a implantação dos sistemas.

Para exprimir a realidade dos agricultores, principalmente aqueles de base familiar, detentores de pequenas áreas destinadas à produção, foram consideradas possíveis perdas (25\%) a cada quatro anos, devido a condições climáticas, enquanto os coeficientes técnicos de preços de venda e produtividade foram estimados pelos menores valores, referenciados na Tabela 2. 
Tabela 2 - Fontes de informações sobre as produtividades e preços de venda em Mato Grosso do Sul, envolvendo as espécies de interesse econômico de sistemas agroflorestais implantados em Dourados, MS. Pesquisa de menor valor de mercado realizada em julho de 2017. Cotação do Dólar em julho de 2020: $1 \$=\mathrm{R} \$ 5,15$.

\begin{tabular}{|c|c|c|c|c|}
\hline \multirow[t]{2}{*}{ Produto } & \multirow{2}{*}{ Produtividade } & \multicolumn{3}{|c|}{ Preço de venda local (Real e Dólar) } \\
\hline & & Fonte & Valor R\$ & Valor \$ \\
\hline Café Arábica & APOMS* & SECAF**/APOMS & $\mathrm{R} \$ 4,50-\mathrm{Kg}$ & $\$ 0.87$ \\
\hline Palmito de Pupunha & APOMS & APOMS & $\mathrm{R} \$ 11,00-\mathrm{Kg}$ & $\$ 2.14$ \\
\hline Banana & $\begin{array}{l}\text { Embrapa Agropecuária } \\
\text { do Oeste }\end{array}$ & APOMS & $\mathrm{R} \$ 1,50-\mathrm{Kg}$ & $\$ 0.29$ \\
\hline Milho & APOMS & APOMS & $\mathrm{R} \$ 1,00-\mathrm{Kg}$ & $\$ 0.19$ \\
\hline Feijão & APOMS & APOMS & $\mathrm{R} \$ 3,50-\mathrm{Kg}$ & $\$ 0.68$ \\
\hline Abacaxi & $\begin{array}{l}\text { Embrapa Informação } \\
\text { Tecnológica }\end{array}$ & SECAF/APOMS & $\mathrm{R} \$ 1,80-\mathrm{Un}$. & $\$ 0.35$ \\
\hline Acerola & Rogério Ritzinger & CEASA-MS*** & $\mathrm{R} \$ 2,50-\mathrm{Kg}$ & $\$ 0.49$ \\
\hline Graviola & $\begin{array}{l}\text { Embrapa Informação } \\
\text { Tecnológica }\end{array}$ & SECAF/APOMS & $\mathrm{R} \$ 2,50-\mathrm{Kg}$ & $\$ 0.49$ \\
\hline Pitanga & $\begin{array}{l}\text { Embrapa Informação } \\
\text { Tecnológica }\end{array}$ & CEASA & $\mathrm{R} \$ 2,50-\mathrm{Kg}$ & $\$ 0.49$ \\
\hline Goiaba & APOMS & APOMS & $\mathrm{R} \$ 2,50-\mathrm{Kg}$ & $\$ 0.49$ \\
\hline
\end{tabular}

*Associação de Produtores Orgânicos de Mato Grosso do Sul; **Serviços de Assistência Técnica para a Agricultura Familiar; ***Central de Abastecimento de Mato Grosso do Sul. Fonte: Autores.

Na Tabela 2, apresenta-se os preços das espécies em entidades do MS, com valor em Real e Dólar, com o intuito de fornecer subsídios para o estudo da viabilidade econômica dos SAFs.

\section{Receitas e custos}

As receitas dos dois sistemas agroflorestais são oriundas das projeções realizadas para a produção de café, palmito de pupunha, milho, feijão, abacaxi, banana, acerola, graviola, pitanga e goiaba.

Os custos com mão de obra se referem à todas as atividades que demandam tempo e que são contabilizadas como homem/dia, cujo valor atual em média é R \$ 70,00. São elas: capina/roçada; amostragem de solo; aplicação de calcário; marcação; plantio e corte de feijão guandu, de feijão de porco e mamona, colheita das sementes de feijão guandu, feijão de porco e mamona e beneficiamento das sementes. As atividades de limpeza, aração e gradagem da área foram contabilizadas como hora/máquina, cujo valor é de $\mathrm{R} \$ 80,00$.

$\mathrm{Na}$ implantação e manutenção de cada cultura, foram necessárias as seguintes atividades: seleção e tratamento de mudas; coveamento; adubação de plantio; plantio; replantio; adubação de cobertura; controle fitossanitário; desbrota; fertilização natural (urina de vaca); poda; desbaste seletivo (sombreamento excessivo); aplicação de fertilizante natural, controle de insetos pragas; prevenção de insetos pragas, controle de gramíneas exóticas; beneficiamento; colheita; debulha manual; ensacamento de sementes; monitoramento e erradicação de cultura. Os transportes internos são computados em hora/maquina $(\mathrm{R} \$ 80,00)$.

Foram considerados os insumos e seus respectivos valores no projeto: mudas ( $\mathrm{R} \$ 0,50$ unid); esterco (R\$100,00 t); adubo fosfatado - termofosfato magnesiano $(\mathrm{R} \$ 250,00 \mathrm{t})$; adubo - compostagem $(\mathrm{R} \$ 110,00 \mathrm{t})$; calda bordalesa $(\mathrm{R} \$ 10,55$ $\mathrm{kg}$ ); óleo de nim (Azadirachta indica A. Juss.) (R\$ 15,00 1); urina de vaca (R\$ 1,00 1); cama de frango (R\$ 100,00 ton) e diesel para perfurador de solo $(\mathrm{R} \$ 3,401)$. 
Entre os insumos, além de adubos, sementes e mudas, está a "munha de carvão" (pó de carvão - resíduo de carvoaria), uma adubação verde de decomposição lenta atuando como matéria orgânica rica em minerais que faz o controle de gramíneas, principalmente braquiária, utilizada no cultivo do palmito de pupunha em sistema agroflorestal.

\section{Resultados}

Os resultados da produção se deram a partir do primeiro ano em ambos os sistemas (Figura 2), devido à colheita das culturas de ciclo rápido.

Figura 2 - Receitas, Custos e Fluxo de caixa ajustado dos sistemas agroflorestais implantados em Dourados, MS e projeções ao longo do tempo.
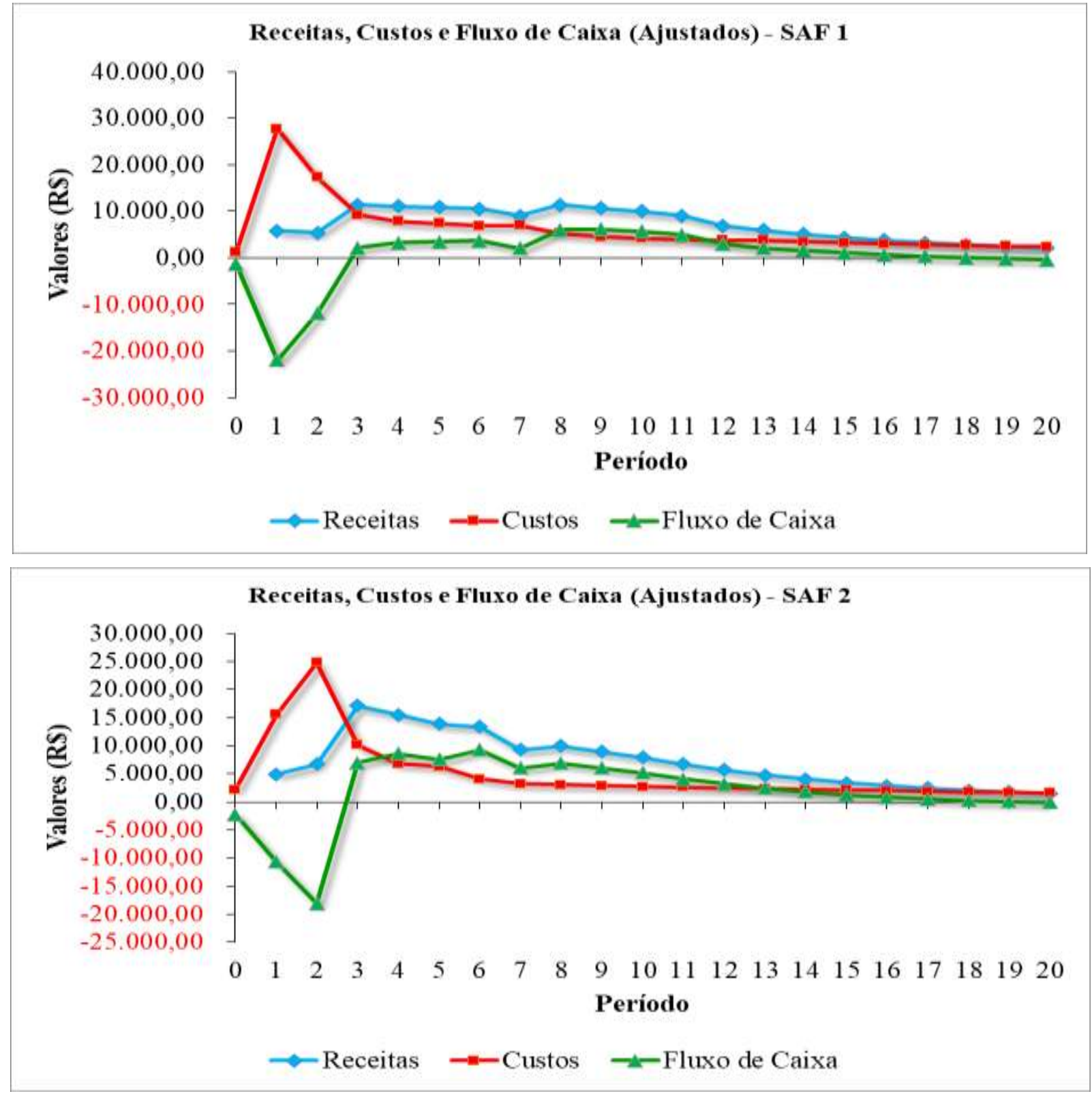

Fonte: Autores.

No entanto, o fluxo de caixa acumulado ajustado foi negativo até o terceiro ano para ambos os SAFs (Figura 2).

Ao se observar o saldo final do projeto ao longo de 20 anos (Tabela 3), percebe-se que o SAF 2 apresentou melhor 
rendimento, contudo ambos apresentaram uma renda líquida positiva no final, com valores de R $\$ 11.018,24$ para o SAF 1 e R $\$$ 40.377,04 para o SAF 2 .

Tabela 3 - Resumo dos valores em R\$ para receitas, despesas e saldo final de sistemas agroflorestais implantados em Dourados, MS e projeções ao longo do tempo. Cotação do Dólar em julho de 2020: 1 \$ = R \$ 5,15.

\begin{tabular}{|c|c|c|c|c|}
\hline \multirow{3}{*}{ Resumo do Projeto } & \multicolumn{4}{|c|}{$\begin{array}{l}\text { Valores (em Reais e Dólar) } \\
\text { Ajustado (período } 20 \text { anos) }\end{array}$} \\
\hline & \multicolumn{2}{|c|}{ SAF 1 } & \multicolumn{2}{|c|}{ SAF 2} \\
\hline & $\mathrm{R} \$$ & $\$$ & $\mathrm{R} \$$ & $\$$ \\
\hline Receitas: & $142.369,85$ & $27.644,63$ & $143,241.60$ & $27.813,90$ \\
\hline Despesas: & $131.351,61$ & $25.505,17$ & $102,864.56$ & $19.973,70$ \\
\hline Saldo Final: & $11.018,24$ & $2.139,46$ & $40.377,04$ & $7.840,20$ \\
\hline
\end{tabular}

Fonte: Autores.

A Tabela 3 apresenta, os valores ajustados no período de 20 anos. A fase mais produtiva do SAF 1 se dá do $3^{\circ}$ ao $11^{\circ}$ ano, que corresponde ao auge da produção cafeeira. Já no SAF 2, essa fase se dá do $3^{\circ}$ ao $6^{\circ}$ ano, devido à produção do palmito de pupunha.

A banana permanece no sistema até o $6^{\circ}$ ano; o abacaxi, até o $3^{\circ}$ ano, enquanto que o milho e o feijão, que constituem o estrato inferior do sistema, estarão apenas nos dois primeiros anos. Já as frutíferas, o café e o palmito permaneceram por 20 anos.

De acordo com a Tabela 4, a taxa mínima de atratividade (TMA) ou taxa de remuneração desejada para o capital próprio investido para ambos os sistemas agroflorestais foi de $6.5 \%$, que é normalmente utilizada nesse tipo de investimento. A taxa interna de retorno do investimento (TIR) no Projeto do SAF 1 foi de $10.57 \%$, enquanto que do SAF 2 foi $24.71 \%$.

Tabela 4 - Indicadores financeiros por 20 anos de sistemas agroflorestais implantados em Dourados, MS e projeções ao longo do tempo.

\begin{tabular}{lll}
\hline Avaliação Financeira & SAF 1 & SAF 2 \\
\hline TMA do Projeto & $6.50 \%$ & $6.50 \%$ \\
TIR do Projeto & $10.57 \%$ & $24.71 \%$ \\
VPL do Projeto & $11,018.24$ & $40,377.04$ \\
Payback simples & 9.0 & 6.0 \\
Payback descontado & 11.0 & 6.0 \\
VAE do Projeto & 999.98 & $3,664.47$ \\
Relação B/C & 1.1 & 1.4 \\
\hline
\end{tabular}

Fonte: Autores.

Na Figura 3 encontram-se os gastos com mão de obra e insumos para os dois SAFs. Para o SAF 1, o gasto com mão de obra é elevado no primeiro ano, representando mais de $80 \%$ dos gastos totais, após este período, tem uma queda e posterior aumento para cerca de $60 \%$ dos gastos totais até o final do projeto. Já o SAF 2, a demanda com mão de obra permanece alta até 
Research, Society and Development, v. 10, n. 4, e47210413682, 2021

(CC BY 4.0) | ISSN 2525-3409 | DOI: http://dx.doi.org/10.33448/rsd-v10i4.13682

o final do projeto, representando quase $90 \%$ dos gastos totais.

Figura 3 - Custos com mão de obra e insumos, durante 20 anos para dois sistemas agroflorestais.

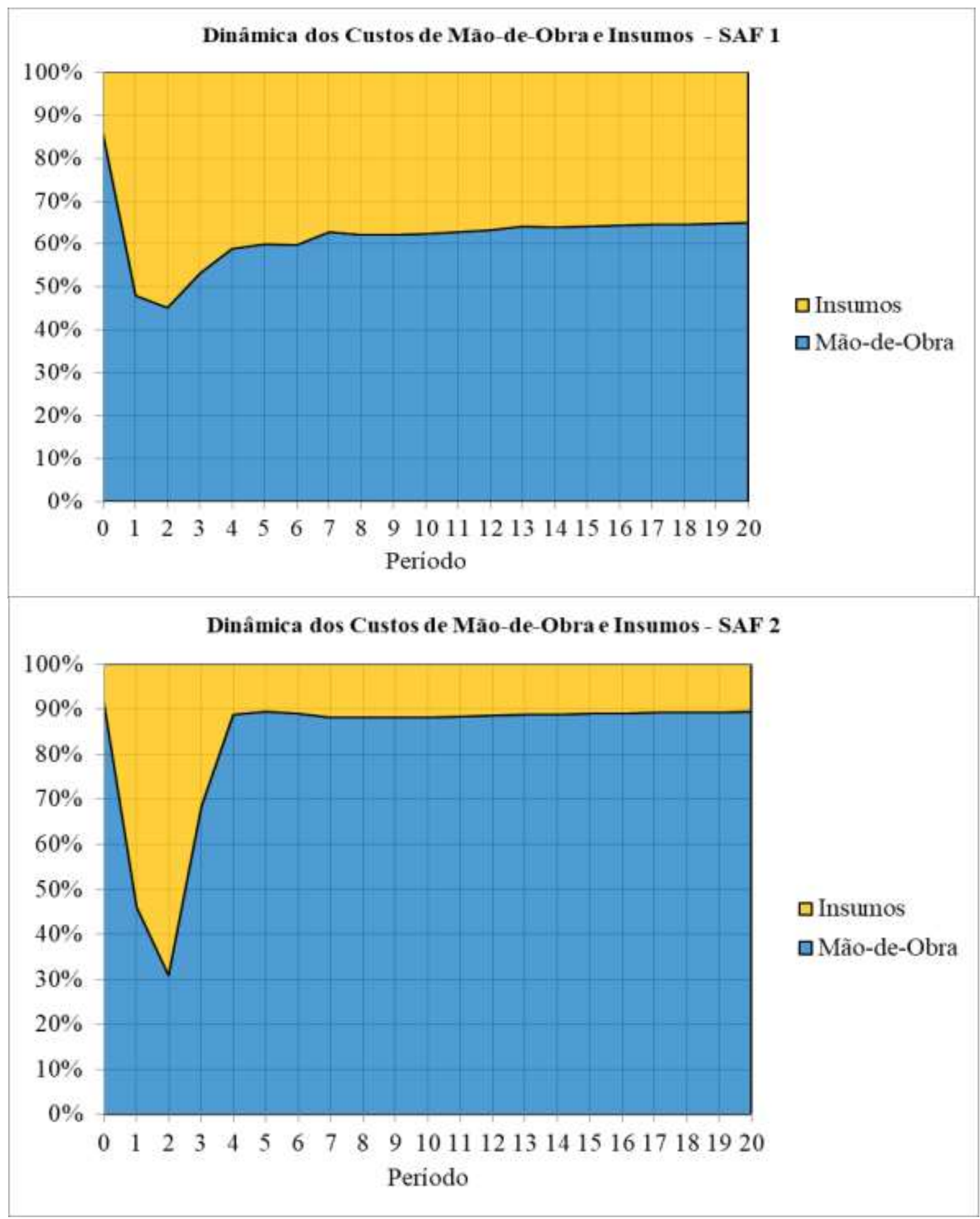

Fonte: Autores.

De acordo com a Figura 4, as espécies ícones de cada SAF que se sobressaíram foram: café e palmito pupunha, seguidas pelas frutíferas. Isso se deve à permanência dessas culturas no sistema ao longo dos 20 anos. 
Figura 4 - Custos e receitas por cultura de dois modelos agroflorestais.

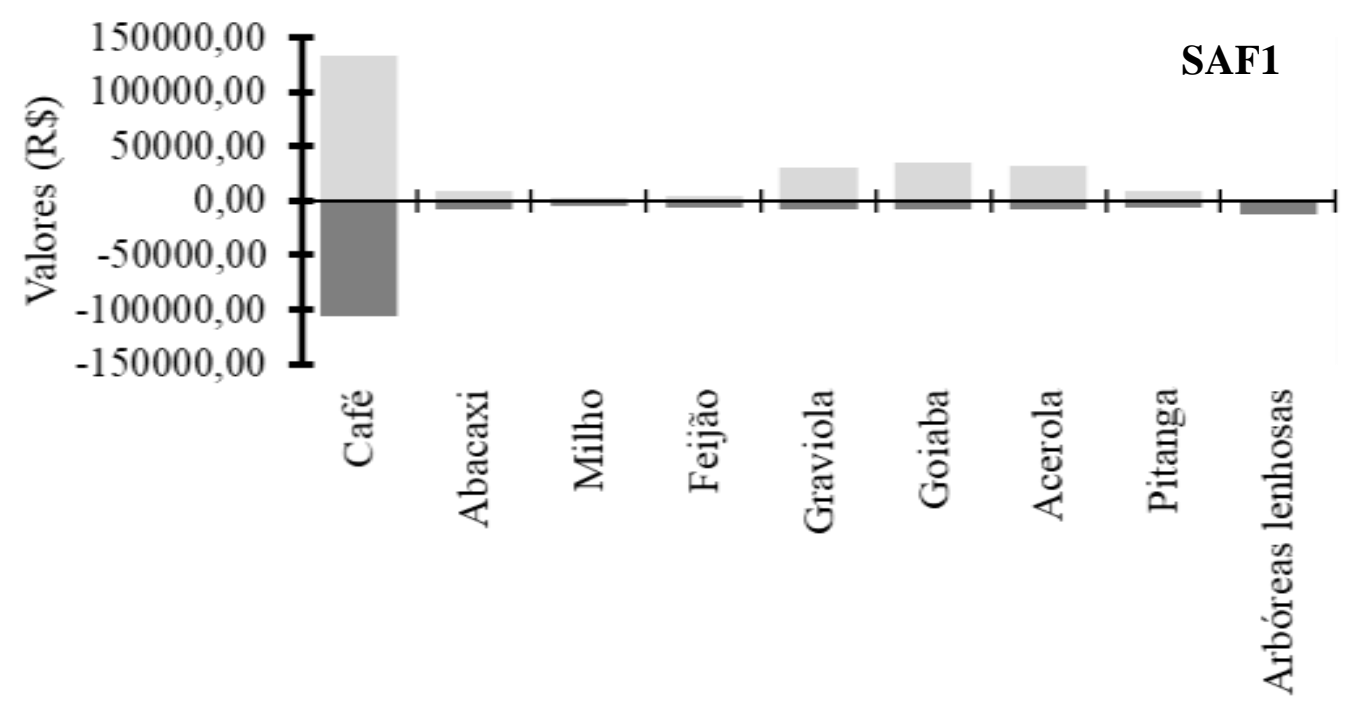

n Custos $\square$ Receitas

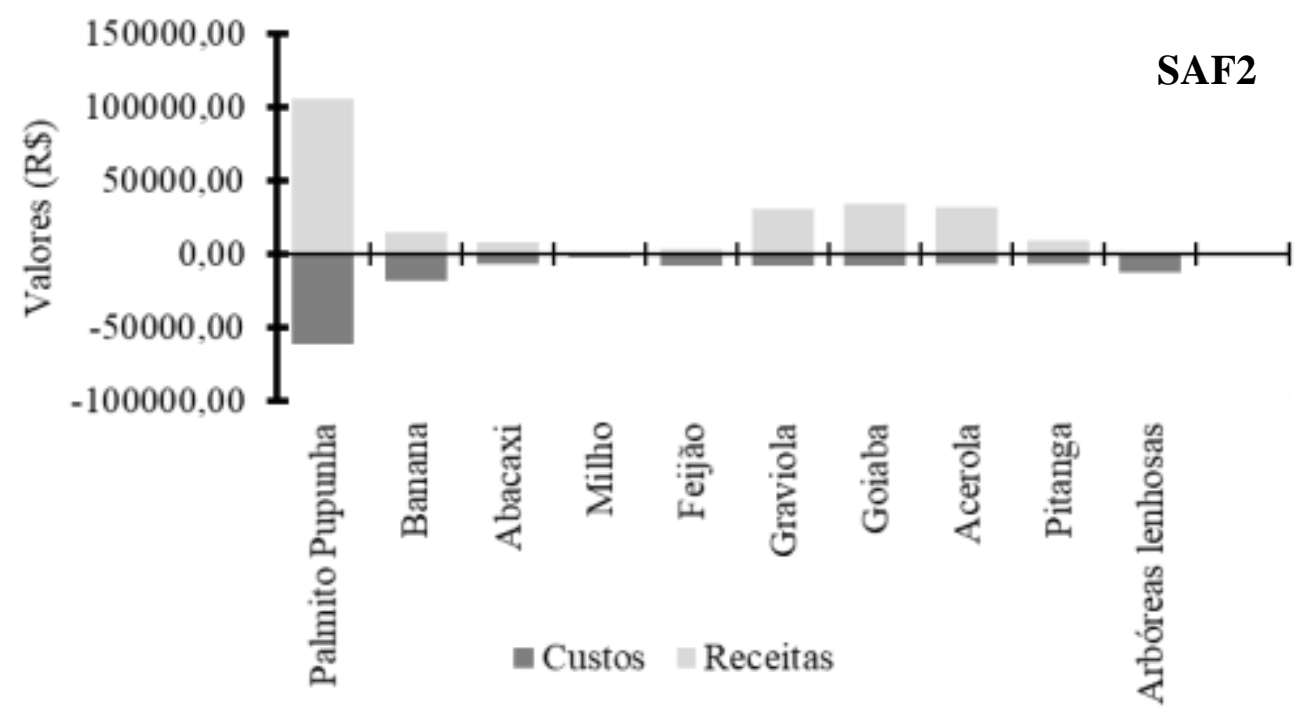

Fonte: Autores.

\section{Discussão}

Os dois sistemas agroflorestais estudados, na projeção realizada para os 20 anos, apontam fluxo de caixa positivo, demostrando que ambos os SAFs são viáveis economicamente e podem ser indicados aos agricultores familiares da região Centro Oeste do Brasil. O SAF 2 apresentou melhor rendimento devido os custos de implantação e manutenção serem um pouco menor de que o SAF1, bem como a inclusão da banana no sistema.

No SAF 1, as despesas foram maiores no primeiro ano, devido aos custos de implantação concomitante de todas as culturas. As receitas foram mais significativas a partir do terceiro ano, apesar de as culturas anuais como feijão, milho e abacaxi gerarem retorno financeiro nos primeiros anos de implantação, elas não são tão representativas. Contudo, estas espécies proporcionam segurança alimentar aos agricultores e podem ser comercializadas, amenizando assim o custo de implantação do sistema conforme já sugerido por Francez e Rosa (2011). 
No SAF 2, os custos permaneceram altos até o segundo ano, devido ao plantio da pupunha que se deu neste período, já que é necessário o sombreamento proporcionado pela mamona, plantada 6 meses antes da pupunha. As receitas iniciaram a partir do primeiro ano, e tiveram seu pico no terceiro ano com o início da colheita das bananas.

Os indicativos financeiros utilizados apontam viabilidade financeira, uma vez que o VPL e TIRM são positivos. Segundo Pereira e Almeida (2008), a TIR pode ser utilizada na comparação entre dois ou mais projetos de investimentos. Nesse caso, o projeto que apresentar o maior valor da TIR será o projeto economicamente mais atraente. Assim, dentre os sistemas analisados no presente estudo, o SAF 2 que apresenta como componente principal para a geração de renda a pupunha, é o mais indicado financeiramente. O payback é o período de recuperação do capital investido, e o SAF 1 demoraria 9 anos, enquanto o SAF 2 transcorreria 6 anos para que o lucro líquido acumulado se iguale ao investimento.

O Valor Anual Equivalente está satisfatório, pois no sistema café (SAF 1) foi cerca de R\$ 999.98 reais e o sistema pupunha-banana (SAF 2) de $\mathrm{R} \$ 3,664.47$ reais, sabendo que a viabilidade do sistema é maior quanto maior o VAE (ArcoVerde \& Amaro, 2010).

$\mathrm{Na}$ análise da Relação Benefício-Custo, observa-se que, utilizando a taxa de desconto de 6,5\%, seus valores correspondem a 1,1 no SAF 1 e 1,4 no SAF 2. Isso significa que, para cada $\mathrm{R} \$ 1,00$ investido, o retorno próximo ao $3^{\circ}$ ano, em ambos os SAFs, isso proporciona uma desmotivação aos agricultores familiares que necessitam de renda a partir do primeiro ano. Assim, sugere-se que além das culturas de ciclo anuais já mencionadas, sejam incluídas hortaliças e cucurbitáceas, como: abóboras e melancias nos sistemas, para que este sejam mais atrativos logo nos primeiros meses do projeto.

A geração de receitas no início da vida dos projetos agroflorestais é fundamental para adesão de mais agricultores no processo. Isso implica que a assistência técnica esteja atenta ao propor modelos de SAFs que sejam adequados para cada região, bem como levar em consideração as demandas de mercado e aptidão do agricultor familiar como já sugerido por Castro Neto et al. (2017).

O SAF 2 demandou mais mão de obra que o SAF1 devido a presença da cultura da banana que demanda uma elevada mão de obra principalmente na fase de produção, uma vez que a colheita da fruta e a maior parte dos tratos culturais realizados na lavoura são feitos manualmente. Ressalta-se que a mão de obra é um fator determinante para tomada de decisão do produtor, pois este avalia ser mais vantajoso selecionar uma cultura que demande menor contratação de mão de obra externa. $\mathrm{O}$ período de implantação é a fase de maior demanda de mão de obra. A partir do $4^{\circ}$ ano, a demanda se estabiliza, pois, cessam as culturas anuais e semiperenes e permanecem apenas as perenes nos agroecossistemas, frutíferas e arbóreas lenhosas. Neves et al. (2014) comentam que as altas despesas da fase inicial de implantação podem ser compensadas com sua diluição ao longo dos anos de exploração do sistema.

A demanda de mão de obra corresponde à despesa mais importante nesses sistemas (Arco-Verde \& Amaro 2014, 2015), embora seja uma atividade realizada pela própria família, representa o custo de oportunidade (Palma et al. 2020). Assim, embora tenha um caráter subjetivo, pois depende do anseio do agricultor, não deve ser negligenciada na hora de elaborar o projeto da SAF e este deve sempre ser elaborado considerando o valor da mão de obra local.

Os insumos tiveram seus picos de utilização no $1^{\circ} \mathrm{e} 2^{\circ}$ ano, respectivamente. Isso se deve à implantação das culturas do café e da pupunha. No SAF 1, a despesa com insumos é maior que o SAF 2, devido à manutenção do café que exige esterco, adubo fosfatado, compostagem, calda bordalesa e óleo de nim, durante todo período de 20 anos.

As espécies ícones de cada SAF que se sobressaíram foram: café e palmito pupunha, seguidas pelas frutíferas. Isso se deve à permanência dessas culturas no sistema ao longo dos 20 anos. Constata-se, ainda, que feijão e milho têm mais despesas que receitas, porém estas culturas são destinadas à subsistência das famílias rurais, além da utilização para alimentação de animais, no caso do milho.

As arbóreas lenhosas (Ipê - Handroanthus heptaphyllus, Guaritá - Astronium graveolens, Aroeira - Myracrodruon 
urundeuva, Canafístula - Peltophorum dubium e Peitos de Pombo - Tapirira guianensis) precisam de desbaste seletivo para evitar o sombreamento excessivo no sistema e a queda na produtividade, como recomendam Araújo et al. (2013). Essa prática tem um custo crescente de mão de obra, já que a cada ano o grau de dificuldade para a poda é maior. Apesar de oferecerem diversos serviços ecossistêmicos (Vasconcellos et al., 2020), em especial nesse projeto como fornecimento de matéria orgânica, sombra, atração à fauna silvestre e abelhas, manutenção da umidade do solo, ciclagem de nutrientes, entre outros, essas espécies arbóreas não são contabilizadas como receitas na planilha, já que não foram selecionadas para extração de madeira. Por essas razões, são necessários mais estudos e elaboração de outros sistemas, testes ou planilhas que incluam o pagamento de serviços ambientais, que, segundo Gandara e Kageyama (2001), "podem ser valorados e convertidos em créditos ambientais, propiciando maior valor à propriedade agrícola”.

Considerando os resultados obtidos nesse trabalho, os SAFs analisados representam grande oportunidade para aos agricultores familiares da região, pois, além dos benefícios ambientais, já amplamente difundidos na literatura (Fifanou et al. 2011; Souza e Piña-Rodrigues, 2013; Dawson et al., 2013; Fernandes et al., 2020), contribuem para a segurança alimentar das famílias agricultoras, bem como à melhoria da qualidade de vida da comunidade local. Além disso, pelo fato da maioria dos insumos serem produzidos nas próprias propriedades e a mão de obra ser familiar, a rentabilidade dos sistemas é maior, como já sugerido por Gao et al. (2014).

Merece destaque ainda o fato de a diversidade dos produtos garantirem maiores possibilidades e flexibilidade ao empreendimento, contribuindo para a diminuição dos riscos (Tremblay et al., 2015).

\section{Conclusão}

A viabilidade financeira, tanto do SAF 1, com café como cultura principal, quanto do SAF 2, com palmito de pupunha e banana (culturas âncoras), foi confirmada, de forma a garantir aos agricultores que, se bem planejados e acompanhados, esses sistemas possuem boas condições para produção de alimentos e geração de renda. Além disso, se tornam soluções possíveis para uma agricultura sustentável, valorizando e utilizando sabiamente os recursos naturais.

O SAF 2 apresentou ser mais rentável, já que seu Valor Presente Líquido é de R\$40,377.04, o payback é de 6 anos e a relação Benefício/Custo de 1,4. Dessa forma, recomenda-se este arranjo para agricultores familiares da região Sul de Mato Grosso do Sul.

Para a tomada de decisão sobre sistemas agroflorestais, recomenda-se um planejamento rigoroso utilizando-se como ferramenta a simples e objetiva planilha AmazonSAF que analisa sistemas de produção de forma bem similar à realidade da agricultura familiar, apesar de não comtemplar os benefícios dos serviços ambientais, tão pouco a economia com o consumo de culturas pelo próprio agricultor. Deve-se levar em consideração a geração de renda desde o primeiro ano de implantação, já que agricultor não pode permanecer sem renda durante muito tempo. Nesse contexto, sugere-se o plantio de culturas de ciclo curto, como as hortaliças e curbubitáceas.

Tornam-se necessários mais estudos sobre pagamento de serviços ambientais prestados por sistemas agroflorestais, a fim de se valorar os serviços ecossistêmicos e sobre a viabilidade financeira desses agroecossistemas, para que haja uma crescente aceitação e confiança de agricultores dispostos a investirem em seu próprio negócio, envolvendo sua família e garantindo melhores condições de vida no campo e transformação social.

\section{Agradecimentos}

Ao Curso de Pós-Graduação em Biologia Geral/Bioprospecção da Universidade Federal da Grande Dourados, CAPES (Coordenação de Aperfeiçoamento de Pessoal de Nível Superior) pela bolsa e CNPQ pelo apoio financeiro Processo $\mathrm{n}^{\circ} 427250$ / 2016-1. 


\section{Referências}

Arai, F. K., Gonçalves, G. G., Pereira, S. B., Comunello, É., Vitorino, A. C., \& Daniel, O. (2010). Espacialização da precipitação e erosividade na Bacia Hidrográfica do Rio Dourados-MS. Engenharia Agrícola, 30 (5), 922-931.

Araújo, L. (2013). Transição agroecológica na comunidade quilombola de Morro Alto, Maquiné-RS. 2013. 20p. Monografia (Tecnológica em Planejamento e Gestão para o Desenvolvimento Rural) Faculdade de Ciências Econômicas, Universidade Federal do Rio Grande do Sul, Santo Antônio da Patrulha.

Arco-Verde, M. F., \& Amaro, G. C. (2010). Oficina sobre Sistemas Agroflorestais - Operação Arco Verde. Manaus: Embrapa Amazônia Ocidental.

Arco-Verde, M. F., \& Amaro, G. C. (2014). Análise financeira de sistemas produtivos integrados. Colombo/PR: Embrapa Florestas. Recuperado de: <https://www.infoteca.cnptia.embrapa.br/handle/doc/1014392>.

Arco-Verde, M. F.; \& Amaro, G. C. (2015). Metodologia para análise da viabilidade financeira e valoração de serviços ambientais em sistemas agroflorestais. In: L. M. Parron; J. R. Garcia; E. B. de Oliveira; G. G. Brown; R. B. Prado (Orgs.); Serviços Ambientais em Sistemas Agrícolas e Florestais do Bioma Mata Atlântica p.335-346. Brasília, DF: Embrapa. <https://www.alice.cnptia.embrapa.br/alice/bitstream/doc/102 4363/1/MarceloAVL ivroServicosAmbientaisCap30.pdf>.

Camargo, G. M., Schlindwein, M. M., Padovan, M. P., \& da Silva, L. F. (2019). Sistemas Agroflorestais Biodiversos: Uma Alternativa Para Pequenas Propriedades Rurais. Revista Brasileira de Gestão e Desenvolvimento Regional, 15 (1), 34-46.

Castro Neto, F. D., Jacovine, L. A. G., Torres, C. M. M. E., Oliveira Neto, S. N. D., Castro, M. M. D., Villanova, P. H., \& Ferreira, G. L. (2017). Balanço de carbono-viabilidade econômica de dois sistemas

Coelho, G. C. (2017). Ecosystem services in Brazilian's southern agroforestry systems. Tropical and Subtropical Agroecosystems, $20(3), 475-492$.

Dawson, I. K., Guariguata, M. R., Loo, J., Weber, J. C., Lengkeek, A., Bush, D., \& Jamnadass, R. (2013). What is the relevance of smallholders' agroforestry systems for conserving tropical tree species and genetic diversity in circa situm, in situ and ex situ settings? A review. Biodiversity and Conservation, 22(2), 301-324. 10.1007/s10531-012-0429-5

Embrapa - Empresa Brasileira de Pesquisa Agropecuária. Centro Nacional de Pesquisa de Solos. (2006). Sistema Brasileiro de Classificação de Solos. Rio de Janeiro: Embrapa. 412p.

Fernandes, S. S. L., Santiago, E. F., Padovan, M. P., Carneiro, L. F., \& de Melo Virginio Filho, E. (2020). Serviços ambientais culturais e de suporte: percepção por agricultores familiares em sistemas agroflorestais do Brasil e Costa Rica. Research, Society and Development, 9 (12). e11691210783e11691210783.

Fifanou, V. G., Ousmane, C., Gauthier, B., \& Brice, S. (2011). Traditional agroforestry systems and biodiversity conservation in Benin (West Africa). Agroforestry systems, 82 (1), 1-13. 10.1007/s10457-011-9377-4

Francez, D. C., \& Rosa, L. S. (2011). Viabilidade econômica de sistemas agroflorestais em áreas de agricultores familiares no Pará, Brasil. Revista de Ciências Agrárias, 54 (2), 178-187.

Gandara, F. B., \& Kageyama, P. Y. (2001). Biodiversidade e dinâmica em sistemas agroflorestais. In: III Congresso Brasileiro de Sistemas Agroflorestais (p. 25). Manaus, AM: Embrapa Amazônia Ocidental.

Gao, J., Barbieri, C., \& Valdivia, C. (2014). A socio-demographic examination of the perceived benefits of agroforestry. Agroforestry systems, 88 (2), 301309. $10.1007 / \mathrm{s} 10457-014-9683-8$

Götsch, E. (1997). Homem e Natureza, cultura na agricultura. (2a ed.), AS-PTA. 22p.

IBGE. (2012). Manual técnico da vegetação brasileira. Série Manuais Técnicos em Geociências. 271p.

Martinelli, G. C., Schlindwein, M. M., Padovan, M. P., Vogel, E., \& Ruviaro, C. F. (2019). Environmental performance of agroforestry systems in the Cerrado biome, Brazil. World Development, 122, 339-348. 10.1016/j.worlddev.2019.06.003.

Miccolis, A., Peneireiro, F. M., Vieira, D. L. M., Marques, H. R., \& Hoffmann, M. R. M. (2019). Restoration through agroforestry: options for reconciling livelihoods with conservation in the Cerrado and Caatinga biomes in Brazil. Experimental Agriculture, 55 (S1), 208-225.10.1017/S0014479717000138.

Molua, E. L. (2005). The economics of tropical agroforestry systems: the case of agroforestry farms in Cameroon. Forest policy and economics, 7(2), 199-211. $10.1016 /$ S1389-9341(03)00032-7.

Mosquera-Losada, M. R., Santiago Freijanes, J. J., Pisanelli, A., Rois, M., Smith, J., den Herder, M., \& Burgess, P. J. (2016). Extent and success of current policy measures to promote agroforestry across Europe. AGFORWARD European Project Policy Report: Bruxelles, Belgium. http://www. agforward.eu/index.php/en/extent-and-success-of-currentpolicy-measures-to-promote-agroforestry-across-europe. html.

Nair, R. P. K. (1993). An introduction to Agroforestry. Kluwer, ICRAF. 499p.

Nair, R. P. K. (2014). Grand challenges in agroecology and land use systems. Frontiers in Environmental Science, 2 (1). 10.3389/fenvs.2014.00001

Nasser, M. D., Tarsitano, M. A. A., Lacerda, M. D., \& Koga, P. S. L. (2012). Análise econômica da produção de café arábica em São Sebastião do Paraíso, Estado de Minas Gerais. Informações Econômicas, 42 (2), 5-12.

Neves, M. C., Moriconi, W., Ramos Filho, L. O., Canuto, J. C., \& Urchei, M. A. (2014). Avaliação econômica da implantação e manutenção de um sistema agroflorestal com cultivo diversificado. In: Congresso da Sociedade Brasileira de Sistemas de Produção, 10. Foz do Iguaçu. 
Research, Society and Development, v. 10, n. 4, e47210413682, 2021

(CC BY 4.0) | ISSN 2525-3409 | DOI: http://dx.doi.org/10.33448/rsd-v10i4.13682

Padovan, M. P., Pereira, Z. V., Nascimento, J. S., Soares, J. A. B., Fernandes, S. S. L., Alves, J., \& Agostinho, P. (2019). Potencial de sistemas agroflorestais biodiversos em processos de restauração ambiental. In: Tayronne de Almeida Rodrigues, João Leandro Neto. (Org.). Competência técnica e responsabilidade social e ambiental nas ciências agrárias. Atena Editora, 127-136.

Padovan, M. P., Pezarico, C. R., Otsubo, A. A. (2014). Sistemas Agroflorestais em bases agroecológicas. Tecnologias para a agricultura familiar, 3, 65-69.

Palma, V. H., Arco-Verde, M. F., Curcio, G. R., Galvão, F., \& Mattos, L. (2020). Análise financeira de sistema agroflorestal (SAF) orgânico do sul do Brasil. Enciclopédia biosfera, 17 (31), 26-39.

Paludo, R., \& Costabeber, J. A. (2012). Sistemas agroflorestais como estratégia de desenvolvimento rural em diferentes biomas brasileiros. Revista Brasileira de Agroecologia, 7 (2), 63-76.

Peneireiro, F. M. (2003). Educação agroflorestal: construindo junto o conhecimento. Anais do II Simpósio de Sistemas Agroflorestais de Sergipe. Aracaju .

Pereira, W. A., Almeida, L.S. (2008). Método Manual para Cálculo da Taxa Interna de Retorno. Revista Objetiva, 4, 38-50.

Pereira, A. S., Shitsuka, D. M., Parreira, F. J., \& Shitsuka, R. (2018). Metodologia da pesquisa científica. UFSM. https://repositorio.ufsm.br/bitstream/handle/1/15824/Lic_Computacao_MetodologiaPesquisaCientifica.pdf?sequence=1.

Sharma, A. B. (2013). An introduction to Agroforestry System. Journal of Natural Sciences, 1 (1), 35-41.

Silva, I. C. (2013). Sistemas Agroflorestais: conceitos e métodos. SBSAF, Itabuna.

Sousa, L. A. S., Galvão, J. R., Pacheco, M. J. B., da Silva Vasconcelos, G., \& de Sousa, A. C. (2020). Chemical attributes of soil in agroforestry systems is conditioned by the management system adopted. Research, Society and Development, 9 (10). e7849108814-e7849108814.

Souza, M. C. S., Piña-Rodrigues, F. C. M. (2013). Desenvolvimento de espécies arbóreas em sistemas agroflorestais para recuperação de áreas degradas na Floresta Ombrófila Densa. Revista Árvore, 37 (1), 89-98

Torralba, M., Fagerholm, N., Burgess, P. J., Moreno, G., \& Plieninger, T. (2016). Do European agroforestry systems enhance biodiversity and ecosystem services? A meta-analysis. Agriculture Ecosystem Environment, 230, 150-161. 10.1016/j.agee.2016.06.002

Tremblay, S., Lucotte, M., Revéret, J. P., Davidson, R., Mertens, F., Passos, C. J. S., \& Romana, C. A. (2015). Agroforestry systems as a profitable alternative to slash and burn practices in small-scale agriculture of the Brazilian Amazon. Agroforestry Systems, 89 (2), 193-204. 10.1007\%2Fs10457-014-9753-y

Udawatta, R. P., Rankoth, L.M., \& Jose, S. (2019). Agroforestry and biodiversity. Sustainability, 11 (10), 2879. 10.3390/su11102879

Vasconcellos, R. C., Beltrão, N. E. S., Martins, S. S., \& de Paula, M. T. (2020). Identificação dos serviços ecossistêmicos na produção agrícola: um estudo em sistemas agroflorestais. Research, Society and Development, 9 (10). e9259109268-e9259109268.

Zomer, R. J., Neufeldt, H., Xu, J., Ahrends, A., Bossio, D., Trabucco, A., \& Wang, M. (2016). Global Tree Cover and Biomass Carbon on Agricultural Land: The contribution of agroforestry to global and national carbon budgets. Scientific reports, 6 (1), 1-12. 10.1038/srep29987

Zomer, R. J., Neufeldt, H., Xu, J., et al., 2016. Global tree cover and biomass carbon on agricultural land: the contribution of agroforestry to global and national carbon budgets. Sci. Rep. 6, 29987. 